\title{
Discover Social Science and Health
}

Research

\section{HPV and Pap testing among white, black, and hispanic women: results from a survey study}

\author{
Ilana M. Ventura ${ }^{1,2} \cdot$ Selena Zhong ${ }^{1,2} \cdot$ Ipek Bilgen $^{1} \cdot$ David Dutwin $^{1} \cdot$ Jane L. Delgado ${ }^{3}$ \\ Received: 20 August 2021 / Accepted: 12 November 2021 \\ Published online: 29 November 2021 \\ (c) The Author(s) 2021 OPEN
}

\begin{abstract}
While the Pap test, HPV vaccine, and HPV test are important tools to promote cervical health, the American Cancer Society (ACS) recently updated its cervical cancer prevention recommendations to give primacy to HPV testing for cervical cancer screening. We investigate how women's health providers view these changes, and the extent to which they have reached American women. To do so, we present results from a novel two-survey study of women's health providers $(\mathrm{N}=558)$ and White, Black, and Hispanic women $(\mathrm{N}=1900)$. We find that a minority of providers report that the HPV test alone is adequate for cervical cancer screening $(18 \%, \mathrm{Cl}=14 \%, 21 \%)$ while $96 \%(\mathrm{Cl}=94 \%, 97 \%)$ indicated that women should regularly have both Pap and HPV screenings. Black $(88 \% \mathrm{Cl}=86 \%, 91 \%)$ and Hispanic $(87 \% \mathrm{Cl}=84 \%, 89 \%)$ women report lower rates of Pap tests compared to White women $(94 \%, \mathrm{Cl}=92 \%, 96 \%)$. Only $35 \%(\mathrm{Cl}=33 \%, 37 \%)$ of women report having had an HPV test, and these levels were similar across groups. White $(29 \%, \mathrm{Cl}=25 \%, 33 \%)$ and Hispanic women $(26 \% \mathrm{Cl}=23 \%, 29 \%)$ report statistically similar levels of provider recommendation for joint Pap-HPV testing, while Black women report statistically lower rates $(22 \%, \mathrm{Cl}=18 \%, 25 \%)$. These patterns hold after controlling for several important socio-demographic variables in logistic regression models. The continued lower rates of Pap tests among Black and Hispanic women, combined with low rates of HPV testing is likely to hinder the ability to reduce cervical cancer incidence and mortality, particularly for Hispanic and Black women.
\end{abstract}

Keywords Health disparities · HPV · Cervical cancer prevention · Women's health

\section{Introduction}

Cervical cancer screening guidelines have changed considerably in the last decade. The 2018 U.S. Preventive Services Task Force (USPSTF) and the 2012 version of the American Cancer Society (ACS) guidelines both recommended the use of Pap tests every three years for women ages $21-29$, and a variation of acceptable alternative tests for those between

Supplementary Information The online version contains supplementary material available at https://doi.org/10.1007/s44155-02100003-9.

$\triangle$ Ilana M. Ventura, ventura-ilana@norc.org | ${ }^{1}$ NORC at The University of Chicago, Chicago, USA. ${ }^{2}$ Department of Sociology, The University of Chicago, Chicago, USA. ${ }^{3}$ Healthy Americas Foundation, Washington, DC, USA. 
30 and $65^{1}[1,2]$-either a Pap test every 3 years, a Human papillomavirus (HPV) test every 5 years, or an HPV-Pap cotest every 5 years. However, the 2020 American Cancer Society's (ACS) guidelines have embraced the HPV test alone every five years as their preferred screening method; the Pap test alone every 3 years was considered acceptable where access to HPV tests are limited [3].

For decades, the Pap test was the standard for cervical cancer screening [4] and has been a valuable screening test for pre-cancerous changes in the cervix, but given HPV's etiological role in cervical cancer [5], screening methods have expanded to include the HPV test and the prophylactic HPV vaccine [4] and has shaped the ACS's decision to adopt the HPV test as the primary screening method. However, there are a multitude of barriers to incorporating HPV testing in medical practice, including a demand for new equipment and laboratories, the implementation of new insurance codes, and the reluctance of providers and patients [6].

Considering recent changes to the American Cancer Society's cervical cancer screening guidelines, it is important to examine how health providers view these recommendations, how providers perceive the utility of the HPV test and the HPV-Pap co-test, as well as women's experiences receiving cervical cancer screening to better understand the current landscape of cervical cancer prevention.

The Pap test has dramatically reduced cervical cancer incidence and mortality [4, 7-9], but recent findings lend support to the HPV test as an effective way to distinguish between women who need increased monitoring and a potential follow-up (tested positive for high-risk HPV) from women who can safely lengthen screening intervals (those who tested negative for high-risk HPV) [10-13]. However, there is also documented evidence of the existence of HPV-negative cervical cancers $[14,15]$ that have a poorer prognosis than HPV-positive cervical cancers [16], and thus, the Pap test is still an important component of cervical cancer prevention.

Even though the American Cancer Society's (ACS) recommendations for cervical cancer screening are intended to apply to all women, results from randomized controlled trials supporting the use of HPV testing did not optimally represent Black and Hispanic women and women of lower socioeconomic status [3]. This is an issue, given that Black and Hispanic women are more likely to experience later diagnoses and higher mortality rates from cervical cancer [17-19] than White women. We also know that Black and Hispanic women use cervical cancer prevention tools and screening methods at different rates [17, 20-25]. In addition, studies show that Black and Hispanic women have less knowledge about the Pap test [26, 27], have limited information on the HPV vaccine, and are significantly less likely to have heard of HPV [25, 28-30] compared to White women. Furthermore, Black and Hispanic patients are less likely to receive a healthcare provider's recommendation for an HPV vaccine or the Pap test [31-34] despite the importance of provider recommendations for participating in cancer screenings [31, 35-38]. Most important of all, Black and Hispanic women are also less likely to have regular healthcare providers compared to White women ${ }^{2}$ [39].

While previous studies have documented that Black and Hispanic women experience structural barriers to accessing Pap tests and HPV vaccinations, less is known about whether Black and Hispanic women also experience similar barriers to receiving an HPV test and the HPV-Pap co-test. Using data from a nationally representative sample of Black, White, and Hispanic women, we aim to test whether there are also disparities in the use of HPV testing and HPV-Pap co-testing, as well as whether disparities persist in Pap testing for Black and Hispanic women.

The 2020 ACS guidelines around cervical cancer screening include lengthened screening intervals for women who are at low risk of developing cervical cancer while continuing to provide monitoring and surveillance of women who test positive for high-risk HPV. These recommendations around HPV testing may serve as guidance for practicing physicians, yet little is known about how physicians perceive these recommendations-especially the ACS's recommended move to primary HPV testing - and whether they have implemented these changes in their own practice. Using data from our physician survey, we describe physicians' perceptions of the HPV test and the HPV-Pap co-test. Moreover, while women's use of the HPV vaccine and Pap test has been well-studied, few studies have explored the prevalence of Pap testing, HPV testing and HPV-Pap co-testing among a representative sample of Hispanic, Black, and White women. Therefore, we also aim to describe the prevalence of HPV testing and HPV-Pap co-testing uptake among a sample of Black, Hispanic

\footnotetext{
12018 USPSTF recommends a Pap test every 3 years, an HPV test every 5 years, or an HPV-Pap co-test every 5 years for women ages 30-66. 2012 ACS guidelines recommend an HPV-Pap co-test every 3 years (preferred) or a Pap test every 3 years (acceptable). Both recommend no screening for women over 65 with normal prior tests and if women are at low risk of cervical cancer.

2 Behavioral Risk Factor Surveillance System (BRFSS) data shows that a larger proportion of Black women $(17.2 \% \mathrm{Cl}=16.1 \%-18.2 \%)$ and Hispanic women $(32.9 \% \mathrm{Cl}=31.6-34.2)$ lack regular healthcare providers than White women $(13.5 \% \mathrm{Cl}=13.2 \%-13.9 \%)[39]$.
} 
and White women, as well as the extent to which women have discussed the Pap and the HPV test with a healthcare provider-especially in comparison to the Pap test, given its longer history-and whether there are sociodemographic differences in discussing the Pap test, the HPV test, and the HPV-Pap co-test with a provider. Studying these issues can help us understand the possible impact of transitioning to the most recent American Cancer Society guidelines for cervical cancer screening, and who it may leave behind.

\section{Methods}

\subsection{Study sample}

The Cervical Cancer Study (CCS) was conducted at NORC at the University of Chicago in 2020 and includes two surveys. The first survey-The Healthcare Providers' survey -is a sample of health care professionals $(N=558)$ that used Dynata's nonprobability health care panel as the sample. The healthcare providers' survey was offered only in English and on the web. While there may be self-selection bias among healthcare professionals in the Dynata Panel since the sample source is an online nonprobability health care panel, the ages of the sampled health care professionals are evenly distributed ( $22 \%$ are between ages $25-45 ; 49 \%$ are between ages $45-59$, and $27 \%$ are aged $60+$ ), indicating a wide range of experience in the women's health field. Gender of respondents was also balanced ( $49 \%$ female, $51 \%$ male). Respondents in the provider study were provided an undisclosed monetary incentive for survey completion.

The second survey-The Women's Survey-is a large, nationally representative study of Hispanic, Black, and White women ages 21-65 $(\mathrm{N}=1900)$ that explores women's experiences receiving available cervical cancer related medical examinations, interventions, and recommendations. The completed interviews included White $(N=534)$, Black $(N=587)$, and Hispanic ( $\mathrm{N}=779$ ) women between the ages of 21-65. The CCS Women's survey was offered in English and Spanish and on the web and phone. Data for the CCS Women's survey used NORC's AmeriSpeak ${ }^{\circledast}$ Panel, ${ }^{3}$ and respondents for the women's study were provided a $\$ 2$ incentive after survey completion.

Sampling strata for this study were based on age, race/ethnicity, education, and gender with the size of the selected sample per sampling stratum determined by the population distribution for each stratum. In addition, sample selection considered expected differential survey completion rates by demographic groups so that the set of panel members with a completed interview for a study is a representative sample of the target population. The AmeriSpeak panel sample for the CCS Women's survey included a supplement from Dynata's nonprobability online opt-in panel for Hispanic respondents ages 21 to $29(\mathrm{~N}=139$, included in the total Hispanic sample of $\mathrm{N}=799)$. To help reduce potential bias in the nonprobability sample, the nonprobability respondent sample was balanced by age and education via quota sampling and was appropriately weighted. The study was approved by NORC's Institutional Review Board (IRB).

\subsection{Measures}

For the physician study, healthcare providers were asked "should women have both Pap and HPV tests as part of regular screening?" (Yes, No) and "Do you believe HPV screening alone is adequate for screening for cervical cancer?" (Yes, No). These questions are novel and developed and tested by the research team for this study. Supplemental analysis also includes the region of residence of the providers.

For the women's study, the key questions women were asked were whether they have ever had a Pap test ${ }^{4}$ and whether they have ever had an HPV test (Yes, No), whether they have discussed each of these medical options with a healthcare provider (Yes, No), whether they have received a recommendation for an HPV-Pap co-test (Yes, No), and the timing

\footnotetext{
${ }^{3}$ Funded and operated by NORC at the University of Chicago, AmeriSpeak ${ }^{\circledR}$ is a probability-based panel designed to be representative of the US household population. Randomly selected US households are sampled using area probability and address-based sampling, with a known, non-zero probability of selection from the NORC National Sample Frame. These sampled households are then contacted by US mail, telephone, and field interviewers (face to face). The panel provides sample coverage of approximately $97 \%$ of the U.S. household population. Those excluded from the sample include people with P.O. Box only addresses, some addresses not listed in the USPS.

Delivery Sequence File, and some newly constructed dwellings. While most AmeriSpeak households participate in surveys by web, noninternet households can participate in AmeriSpeak surveys by telephone. Households without conventional internet access but having web access via smartphones are allowed to participate in AmeriSpeak surveys by web. For more information, see [40].

4 The question "Have you ever had a Pap test?" was drawn from the Behavioral Risk Factor Surveillance System (BRFSS) to maintain statistical validity. Our results for this question (see Table 2) fall within the $95 \%$ confidence interval range for BRFSS 2018 -the last time this question was asked in BRFSS and available on the BRFSS Web Enabled Analysis Tool. See [41] for questionnaire.
} 
Table 1 Provider Perceptions of Pap and HPV Testing

\begin{tabular}{lll}
\hline Variable & Mean & $(95 \% \mathrm{Cl})$ \\
\hline $\begin{array}{l}\text { Should women have both Pap and HPV tests as part of } \\
\text { regular screening? }(1=\text { yes, } 0=\text { no })\end{array}$ & 0.96 & $(0.94,0.97)$ \\
$\begin{array}{l}\text { Do you believe HPV screening alone is adequate for } \\
\text { screening for cervical cancer? }(1=\text { yes, } 0=\text { no) }\end{array}$ & 0.18 & $(0.14,0.21)$ \\
\hline
\end{tabular}

of their last Pap test ( $<3$ years ago, $3+$ years ago). Specifically, women were asked "When was your last Pap test?" and answers were coded into two categories: less than 3 years ago $(=1)$ and 3 years ago or more $(=0)$. The exact wordings for the remainder of these questions are presented in the results section alongside the findings. Women were also asked to report their race/ethnicity, age, educational level, marital status, employment status, household composition, and income.

All questions were pretested ( $N=48$ for the Women's Survey and $N=25$ for the Healthcare Provider Survey) and reviewed before the data collection started.

\subsection{Statistical analysis}

Statistical analysis was carried out using SAS software 9.4. We first tabulated the proportion of providers who endorsed the ACS's recommendation that HPV testing alone is adequate for cervical cancer screening and the proportion who believed that women should have the HPV-Pap co-test. We then tabulated the proportion of women who had ever had an HPV test, a Pap test, and a provider recommendation for an HPV-Pap co-test.

Differences among Black, Hispanic, and White women in receiving a Pap test, an HPV test, and a provider recommendation for each of the cervical cancer prevention tools were determined by using $95 \%$ Confidence Intervals $(\mathrm{Cl})$ on the means. We used logistic regression analyses (using SAS PROC LOGISTIC procedure) to determine the likelihood of reporting having had a Pap test, an HPV test, and a provider recommendation for an HPV-Pap co-test among Black, Hispanic and White women from various age groups (see Table 3 for model results). For all the regression models, covariates include race/ethnicity, age, education, household income, and having a provider. All analyses were weighted to represent the general population of Black, Hispanic, and White women.

\section{Results}

\subsection{Provider reports}

An overwhelming majority of healthcare providers $(96 \%, \mathrm{Cl}=0.94,0.97)$ reported that women should have both the Pap and HPV tests as part of their regular healthcare screening, and only $18 \%(\mathrm{Cl}=0.14,0.21)$ reported that an HPV test alone was adequate for screening for cervical cancer (Table 1). This reveals that there is a disjuncture between healthcare providers' perceptions about the adequacy of the HPV test alone in screening for cervical cancer and the 2020 recommendations of the American Cancer Society.

\subsection{Women's reports}

Among our sample of women, an overwhelming majority of women reported having talked with a provider about a Pap test (88\%) and have ever had a Pap test (92\%) (Table 2). These results reveal that Pap tests have had an impressive reach as a cervical cancer screening method. Given the well-documented importance of Pap tests in reducing cervical cancer incidence and mortality [8], the widespread promotion and use of Pap tests can be understood as a success in public health interventions in women's health.

While most women have talked with a healthcare provider about Pap tests and have had a Pap test, there are nonetheless differences in these outcomes. After controlling for having a provider, age, education and household income, logistic regression models (Table 3 ) show that Black $(\mathrm{OR}=0.48[\mathrm{Cl}=0.29,0.78])$ and Hispanic $(\mathrm{OR}=0.56[\mathrm{Cl}=0.36,0.86])$ women have lower odds of having had a Pap test in their lifetime than White women, consistent with previous research $[26,27]$. 


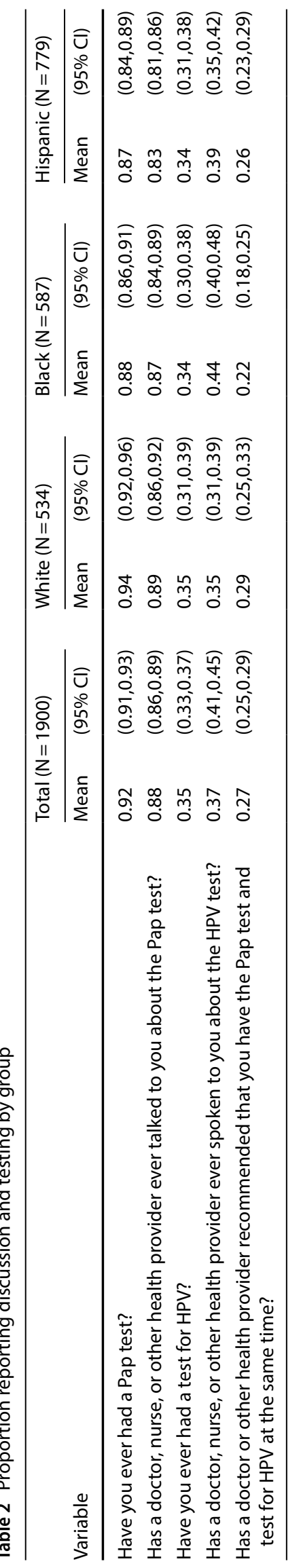


Table 3 Logistic regression: estimating Pap, HPV and HPV-Pap testing recommendations

\begin{tabular}{|c|c|c|c|c|}
\hline & & \multicolumn{3}{|l|}{ OR $(95 \% \mathrm{Cl})$} \\
\hline & & $\begin{array}{l}\text { Estimating Pap Test } \\
(\mathrm{N}=1889)\end{array}$ & $\begin{array}{l}\text { Estimating HPV Test } \\
(\mathrm{N}=1882)\end{array}$ & $\begin{array}{l}\text { Estimating Provider Recom- } \\
\text { mendation for HPV-Pap Co-test } \\
(\mathrm{N}=1878)\end{array}$ \\
\hline \multirow[t]{2}{*}{ Group } & Black, NH vs White, $\mathrm{NH}$ & $0.48(0.29,0.78)^{*}$ & $1.32(0.74,0.99)$ & $0.98(0.51,0.70)^{*}$ \\
\hline & Hispanic vs White, NH & $0.56(0.36,0.86)^{*}$ & $1.29(0.76,0.99)$ & $1.20(0.68,0.90)$ \\
\hline Provider Yes vs No & & $4.59(3.06,6.89)^{*}$ & $2.33(1.18,1.66)^{*}$ & $2.73(1.28,1.87)^{*}$ \\
\hline \multirow[t]{2}{*}{ Age group } & $21-30$ vs $46+$ & $0.15(0.10,0.24)^{*}$ & $2.64(1.56,2.03)^{*}$ & $3.66(2.05,2.74)^{*}$ \\
\hline & $31-45$ vs $46+$ & $0.57(0.34,0.95)^{*}$ & $3.55(2.25,2.83)^{*}$ & $4.05(2.45,3.15)^{*}$ \\
\hline \multirow[t]{3}{*}{ Education } & $\mathrm{BA}+\mathrm{vs}$ less than $\mathrm{HS}$ & $3.09(1.50,6.37)^{*}$ & $2.17(0.88,1.38)$ & $2.42(0.89,1.47)$ \\
\hline & HS graduate vs less than HS & $1.16(0.62,2.17)$ & $1.26(0.51,0.80)$ & $1.54(0.56,0.93)$ \\
\hline & Some college vs less than HS & $1.71(0.89,3.28)$ & $2.08(0.86,1.34)$ & $2.34(0.87,1.43)$ \\
\hline \multirow[t]{3}{*}{ Household income } & $\$ 100 k+v s<\$ 30 k$ & $1.41(0.66,2.98)$ & $1.95(1.00,1.4)$ & $2.48(1.21,1.73)^{*}$ \\
\hline & $\$ 30 k-<\$ 60 k$ vs $<\$ 30 k$ & $0.88(0.56,1.37)$ & $1.58(0.92,1.20)$ & $1.63(0.90,1.21)$ \\
\hline & $\$ 60 k-<\$ 100 k$ vs $<\$ 30 k$ & $0.64(0.38,1.09)$ & $1.36(0.75,1.01)$ & $1.59(0.82,1.14)$ \\
\hline
\end{tabular}

${ }^{*} \mathrm{P}<0.05$

Table 4 Last Pap test less than three years ago

\begin{tabular}{lll}
\hline & \multicolumn{2}{l}{ Total $(\mathrm{n}=1900)$} \\
\cline { 2 - 3 } Variable & Mean & $(95 \% \mathrm{Cl})$ \\
\hline All & 0.76 & $(0.73,0.8)$ \\
White & 0.74 & $(0.69,0.79)$ \\
Black & 0.81 & $(0.77,0.86)$ \\
Hispanic & 0.80 & $(0.76,0.85)$ \\
\hline
\end{tabular}

However, among women who have ever had a Pap test in their lifetime, a larger proportion of Black and Hispanic women had more recent Pap tests. $81 \%$ of Black women $(\mathrm{Cl}=77 \%, 86 \%)$ and $80 \%$ of Hispanic women $(\mathrm{Cl}=76 \%, 85 \%)$ had a Pap test less than three years ago, while only $74 \%$ of White women $(\mathrm{Cl}=69 \%, 79 \%)$ had a Pap test within the past three years (Table 4).

In contrast to the near universal adoption of Pap tests, we found that only $37 \%$ of women have talked with their provider about an HPV test, and only 35\% have ever had an HPV test. These results show that many women are neither receiving an HPV test nor having discussions with their healthcare providers about this important screening test.

While we found that Black and Hispanic women were less likely to have ever had a Pap test than White women, our logistic regression analysis estimating having had an HPV test (Table 3 ) revealed that there were no statistical differences between Black, Hispanic, and White women in HPV test uptake. We found that women with a regular healthcare provider were twice as likely to have had an HPV test compared with those who do not have a regular healthcare provider $(\mathrm{OR}=2.33, \mathrm{Cl}=[1.18,1.66])$. We also found that younger women were more likely to have had an HPV test than older women $(21-30$ vs $46+\mathrm{OR}=2.64[\mathrm{Cl}=1.56,2.03] ; 31-45$ vs $46+\mathrm{OR}=3.55[\mathrm{Cl}=2.25,2.83])$.

Although the percentage of women who have ever talked with a provider about an HPV test is small, the proportion of women who have had an HPV-Pap co-test recommendation from a provider was even smaller, with only $27 \%(\mathrm{Cl}=25 \%$, 29\%) of women reporting that they have received a provider recommendation for having the Pap and HPV test at the same time. Yet in our physician survey, we found that $96 \%$ of providers believed that women should have the HPV-Pap cotest as part of their regular screening. This could suggest that even though providers believe in the co-test, they may not be discussing this option with their patients, or that some women were not aware that one sample is used for both tests.

Descriptive statistics show that White $(29 \%, \mathrm{Cl}=25 \%, 33 \%)$ and Hispanic women $(26 \%, \mathrm{Cl}=23 \%, 29 \%)$ report statistically similar levels of receiving provider recommendation for the HPV-Pap co-test, while Black women report statistically lower rates $(22 \%, \mathrm{Cl}=18 \%, 25 \%)$. Our logistic regression analysis (Table 3 ) shows that, even when controlling for covariates, Black women were still less likely than White and Hispanic women to receive a provider recommendation for an HPV-Pap co-test. In addition, we found that women who have a regular healthcare provider were more likely to receive 
a recommendation for the HPV-Pap co-test than women who do not $(\mathrm{OR}=2.73, \mathrm{Cl}=[1.28,1.87])$. Younger women were more likely to receive an HPV-Pap co-test recommendation than older women (21-30 vs $46+\mathrm{OR}=3.66, \mathrm{Cl}=[2.05,2.74]$; $31-45$ vs $46+\mathrm{OR}=4.05, \mathrm{Cl}=[2.45,3.15])$, and the highest income group $(\$ 100 \mathrm{k}+)$ is statistically significantly more likely than the lowest income group $(<\$ 30 \mathrm{k})$ to have had an HPV-Pap co-test recommendation $(\mathrm{OR}=2.48, \mathrm{Cl}=[1.21,1.73])$.

\section{Discussion}

The Pap test has been used for cervical cancer screening since the first half of the twentieth century and can detect cell changes on the cervix at risk of becoming cervical cancer if left untreated [4, 42]. It appears that public health campaigns around the Pap test were efficacious, as demonstrated by the fact that not only have most women had a Pap test in their lifetime, but most importantly, that cervical cancer incidence and mortality rates have significantly decreased in recent years. These benefits, however, have not reached all women [20-22]. Our findings, consistent with previous research [23], reveal that in spite of the increased access to the Pap test, Black and Hispanic women are still less likely to have ever had a Pap test or to have spoken with a provider about a Pap test.

At the same time, we also find that among women who have ever had a Pap test, a larger proportion of Black and Hispanic women have had a Pap test within the past three years than White women; yet Black and Hispanic women are still more likely to be diagnosed later with, and to die of, cervical cancer [17-19]. While our data cannot directly address why this is so, despite the more recent Pap tests, Black and Hispanic women have worse cervical cancer outcomes than White women. The literature provides several possible explanations: First, having ever received a Pap test may not address the critical access issue of having a regular source of care. Black and Hispanic women are less likely to have regular healthcare providers compared to White women [39], so if they had an abnormal cervical screening test, they may not receive adequate follow-up care, such as a referral for or follow-up with a colposcopy; indeed, research finds that Black and Hispanic individuals are less likely to have follow-up appointments for test results than White individuals [43]. Second, Black and Hispanic women have lower HPV vaccination rates compared to White Women [44]. The lower HPV vaccination rates among Black and Hispanic women may also contribute to higher rates of cervical cancer among these women.

Previous studies on cervical cancer prevention have mostly focused on Pap tests, HPV vaccinations, and discussions with providers about Pap tests and HPV vaccinations, yet little is known about women's experiences with HPV testing, HPV-Pap co-testing, and discussions with providers about HPV tests. Our study attempts to address this gap in the literature by describing the experiences of women with HPV testing and HPV-Pap co-testing, as well as providers' perceptions of these tests. We found that women's participation in HPV testing is quite limited; while most women have had a Pap test, less than half of the women surveyed have had an HPV test or discussed this test with their healthcare providers. That HPV is a sexually transmitted infection (STI) may help explain this finding; women may not feel comfortable discussing an STI, even one that is as common as HPV, with their healthcare providers. Further, research has shown that even patients seen at STI clinics are sometimes unaware of which tests are performed [45]. While rates of cervical cancer incidence and mortality are higher for Black and Hispanic women compared to White women $[20-22,24,25,46,47]$, we find that Black and Hispanic women are no more likely than White women to have had an HPV test in their lifetimes.

While the new ACS guidelines promote the primary HPV test for cervical cancer screening, findings from the Provider Survey show that only $18 \%$ of physicians believe that the HPV test alone is adequate for cervical cancer screening. This may explain why HPV test uptake is universally low for all women. Additionally, while the HPV DNA test was first approved in 2011 to be specifically used alongside the Pap test, the primary HPV test was not approved by the FDA until 2020-which is quite a recent development [48]. Unlike the first iteration of the HPV test and those used in co-testing, the newer primary HPV test is able to detect more HPV genotypes-including high risk types 16 and 18 [49].We hypothesize that some physicians may not want to depend solely on HPV testing for cancer screening, as these tests are mainly used to determine follow-ups with a colposcopy or Pap test, depending on the HPV type [48]. Additionally, as HPV is quite common and has no treatment-and for many women with lower-risk HPV types goes away on its own without significant health issues [50] - HPV testing may pose risk for over-treatment. However, long-term infections with high-risk forms of HPV can cause cervical cancer and genital warts [51].

Supplementary Table S1 shows that provider opinion on the adequacy of HPV testing is higher in the Northeast and West, compared to the Midwest and South regions of the U.S. While regional variation may explain some of the differences in patient screening, more research is necessary to understand these differences. Further research should 
study HPV testing and co-testing availability by different geographic levels-especially for rural clinics-as well as how such availability may be spatially correlated with underserved populations. However, we also found that $96 \%$ of providers believe that an HPV-Pap co-test, where typically one sample is taken and tested for both the HPV DNA and precancerous cells, is important. It may be the case that providers are indeed performing an HPV test at the same time as a Pap test but are failing to communicate this to their patients. Further research is needed to determine whether the low reports of HPV testing among women stems from a lack of access to this test, or whether women have had them but understand them as Pap tests instead of as an HPV-Pap combined test.

\title{
4.1 Limitations
}

One limitation of this study is that the survey is based on women's self-reports of their healthcare experiences, which may be subject to retrospective bias. Having access to medical records or insurance reimbursement could strengthen these self-reports. However, even though these are self-reports, they reflect women's perceptions of their healthcare encounters and experiences with each of the different cervical cancer prevention tools, and these perceptions may inform women's healthcare seeking behavior.

While we surveyed physicians' beliefs about the adequacy of the HPV test alone as a cervical cancer screening method, we did not measure how often physicians performed these screening tests with their patients or whether they reported discussing this option with their patients. However, physicians' beliefs could inform actual clinical practices and if providers do not believe that HPV testing alone is adequate for cervical cancer screening, they may not recommend this testing option to their patients. Because our survey also includes women's reports of the use of the HPV test and their reports of having received a provider recommendation for the use of these tests, we are able to confirm that both only a minority of physicians believe in the benefit of HPV testing alone and only a minority of women have had an HPV test or discussed this option with a healthcare provider, providing support that physicians' beliefs could be a proxy for actual clinical behavior.

Additionally, while this study has shown that disparities persist for Black and Hispanic women around the use of the Pap test and receiving an HPV-Pap co-testing recommendation from a provider, it did not include women of other backgrounds such as Asian American, Middle Eastern, and Native American. To fully understand how access to and discussions with providers about cervical cancer prevention strategies affect all women, future studies should expand their sample to include women from other backgrounds.

\section{Conclusion}

With changing cervical cancer screening guidelines, it is imperative that further research evaluate how these new guidelines impact Black, Hispanic, and White women's experiences with cervical cancer screening and whether they further reduce cervical cancer incidence and mortality rates. While the Pap test, the HPV vaccine, and the HPV test are important tools to prevent cervical cancer, Black and Hispanic women still bear the burden of higher cervical cancer rates and mortality. Therefore, we encourage public health experts to continue to promote cervical cancer screenings through outreach-including through web, social media, TV, print media, and radio messages - to all women, and especially to Black and Hispanic women.

\begin{abstract}
Acknowledgements This work is supported in part by Hologic, Inc., who did not have any role in study design collection, analysis, interpretation of data, writing the report or the decision to submit the report for publication. Jane L. Delgado supervised the study and commissioned the project from NORC on behalf of the Healthy Americas Foundation and National Alliance for Hispanic Health. Jane Delgado is Vice Chair of Lovelace Biomedical Research Institute, on the Board of Governors of Argonne National Labs, and President and CEO of Healthy Americas Foundation and the National Alliance for Hispanic Health. NORC Institutional Review Board IRB Protocol number 20.11.16.
\end{abstract}

Authors' contributions Methodology: IB, DD; Formal analysis and investigation: IV; Writing—original draft preparation: IV, SZ; Writing-review and editing: IB, DD, JD; Funding acquisition: IB, DD; Supervision: JD. IV and SZ wrote the main manuscript text and IV prepared Tables 1-4. IB and DD supervised the methodology. JD supervised and funded the project. All authors reviewed, read and approved the final manuscript.

Funding This research was conducted by NORC researchers in collaboration with the Healthy Americas Foundation, who funded this study. Healthy Americas Foundation receives partial financial support from Hologic, Inc. 
Data availability The datasets generated during and/or analyzed during the current study are available from the corresponding author upon reasonable request.

Code availability Not applicable.

\section{Declarations}

Competing interests This work is supported in part by Hologic, Inc., who did not have any role in the study design, collection, analysis, interpretation of data, writing the report, or the decision to submit the report for publication. Jane L. Delgado supervised the study and commissioned the project from NORC on behalf of the Healthy Americas Foundation and the National Alliance for Hispanic Health. Jane Delgado is Vice Chair of Lovelace Biomedical Research Institute, on the Board of Governors of Argonne National Labs, and is the President and CEO of Healthy Americas Foundation and the National Alliance for Hispanic Health. Questionnaire and methodology approval was granted by the Institutional Review Board (IRB) at NORC at the University of Chicago. The research was carried out following the guidelines of the NORC IRB, in accordance with U.S. Department of Health and Human Services regulations. NORC's IRB is registered with the Federal Office for Human Research Protections. Informed consent was obtained from all individual participants included in the survey.

Open Access This article is licensed under a Creative Commons Attribution 4.0 International License, which permits use, sharing, adaptation, distribution and reproduction in any medium or format, as long as you give appropriate credit to the original author(s) and the source, provide a link to the Creative Commons licence, and indicate if changes were made. The images or other third party material in this article are included in the article's Creative Commons licence, unless indicated otherwise in a credit line to the material. If material is not included in the article's Creative Commons licence and your intended use is not permitted by statutory regulation or exceeds the permitted use, you will need to obtain permission directly from the copyright holder. To view a copy of this licence, visit http://creativecommons.org/licenses/by/4.0/.

\section{References}

1. Saslow D, Solomon D, Lawson HW, Killackey M, Kulasingam SL, Cain J, et al. American Cancer Society, American Society for Colposcopy and Cervical Pathology, and American Society for Clinical Pathology screening guidelines for the prevention and early detection of cervical cancer. CA Cancer J Clin. 2012;62:147-72.

2. US Preventive Services Task Force. Screening for cervical cancer: US preventive services task force recommendation statement. JAMA. 2018;320:674-86.

3. Cervical cancer screening for individuals at average risk: 2020 guideline update from the American Cancer Society-Fontham-2020 CA: A Cancer Journal for Clinicians—Wiley Online Library. https://acsjournals.onlinelibrary.wiley.com/doi/https://doi.org/10.3322/caac. 21628. Accessed 16 Jul 2021.

4. Boone JD, Erickson BK, Huh WK. New insights into cervical cancer screening. J Gynecol Oncol. 2012;23:282-7.

5. Castellsagué X. Natural history and epidemiology of HPV infection and cervical cancer. Gynecol Oncol. 2008;110:S4-7.

6. Marcus JZ, Cason P, Downs LSJ, Einstein MH, Flowers L. The ASCCP cervical cancer screening task force endorsement and opinion on the American Cancer society updated cervical cancer screening guidelines. J Low Genit Tract Dis. 2021;25:187-91.

7. Lazcano-Ponce E, Palacio-Mejia LS, Allen-Leigh B, Yunes-Diaz E, Alonso P, Schiavon R, et al. Decreasing cervical cancer mortality in mexico: effect of papanicolaou coverage, birthrate, and the importance of diagnostic validity of cytology. Cancer Epidemiol Biomarkers Prev. 2008;17:2808-17.

8. Yang DX, Soulos PR, Davis B, Gross CP, Yu JB. Impact of widespread cervical cancer screening: number of cancers prevented and changes in race-specific incidence. Am J Clin Oncol. 2018;41:289-94.

9. Mählck CG, Jonsson H, Lenner P. Pap smear screening and changes in cervical cancer mortality in Sweden. Int J Gynecol Obstet. 1994;44:267-72.

10. Wright TCJ. Cervical cancer screening in the 21st century: is it time to retire the PAP smear? Clin Obstet Gynecol. 2007;50:313-23.

11. Clavel C, Cucherousset J, Lorenzato M, Caudroy S, Nou JM, Nazeyrollas P, et al. Negative human papillomavirus testing in normal smears selects a population at low risk for developing high-grade cervical lesions. Br J Cancer. 2004;90:1803-8.

12. Gage JC, Schiffman M, Katki HA, Castle PE, Fetterman B, Wentzensen N, et al. Reassurance against future risk of precancer and cancer conferred by a negative human papillomavirus test. J Natl Cancer Inst. 2014. https://doi.org/10.1093/jnci/dju153.

13. Schneider A, Hoyer H, Lotz B, Leistritza S, Kühne-Heid R, Nindl I, et al. Screening for high-grade cervical intra-epithelial neoplasia and cancer by testing for high-risk HPV, routine cytology or colposcopy. Int J Cancer. 2000;89:529-34.

14. Liu Y, Xu Y, Jiang W, Ji H, Wang Z-W, Zhu X. Discovery of key genes as novel biomarkers specifically associated with HPV-negative cervical cancer. Mol Therapy Methods Clin Dev. 2021;21:492-506.

15. Nicolás I, Marimon L, Barnadas E, Saco A, Rodríguez-Carunchio L, Fusté P, et al. HPV-negative tumors of the uterine cervix. Mod Pathol. 2019;32:1189-96.

16. Rodríguez-Carunchio L, Soveral I, Steenbergen R, Torné A, Martinez S, Fusté $P$, et al. HPV-negative carcinoma of the uterine cervix: a distinct type of cervical cancer with poor prognosis. BJOG Int J Obstet Gynaecol. 2015;122:119-27.

17. National Cancer Institute. Surveillance, epidemiology, and end results program (SEER). https://seer.cancer.gov/. Accessed 15 Feb 2021.

18. Cancer and Hispanic Americans-The Office of Minority Health. https://minorityhealth.hhs.gov/omh/browse.aspx?lvl=4\&lvlid=61. Accessed 27 May 2021.

19. Cancer and African Americans - The Office of Minority Health. https://minorityhealth.hhs.gov/omh/browse.aspx?lvl=4\&lvlid=16. Accessed 27 May 2021.

20. Adams SA, Fleming A, Brandt HM, et al. Racial disparities in cervical cancer mortality in an African American and European American cohort in South Carolina. J S C Med Assoc. 2009;105(7):237-44. 
21. Eng TY, Chen T, Vincent J, Patel AJ, Clyburn V, Ha CS. Persistent disparities in hispanics with cervical cancer in a major city. J Racial Ethn Health Disparities. 2017;4:165-8.

22. Musselwhite LW, Oliveira CM, Kwaramba T, de Paula PN, Smith JS, Fregnani JH, et al. Racial/ethnic disparities in cervical cancer screening and outcomes. Acta Cytol. 2016;60:518-26.

23. Bazargan M, Bazargan SH, Farooq M, Baker RS. Correlates of cervical cancer screening among underserved Hispanic and African-American women. Prev Med. 2004;39:465-73.

24. Gelman A, Miller E, Schwarz EB, Akers AY, Jeong K, Borrero S. Racial disparities in human papillomavirus vaccination: does access matter? J Adolesc Health. 2013;53:756-62.

25. Gelman A, Nikolajski C, Schwarz EB, Borrero S. Racial disparities in awareness of the human papillomavirus. J Womens Health. 2011;20:1165-73.

26. Breitkopf $\mathrm{CR}$, Pearson HC, Breitkopf DM. Poor knowledge regarding the pap test among low-income women undergoing routine screening. Perspect Sex Reprod Health. 2005;37:78-84.

27. Chan DNS, So WKW. A systematic review of the factors influencing ethnic minority women's cervical cancer screening behavior: from intrapersonal to policy level. Cancer Nurs. 2017;40:E1.

28. Bowen DJ, Weiner D, Samos M, Canales MK. Exploration of New England Native American women's views on human papillomavirus (HPV), testing, and vaccination. J Racial Ethn Health Disparities. 2014;1:45-51.

29. Ford JL. Racial and ethnic disparities in human papillomavirus awareness and vaccination among young adult women. Public Health Nurs. 2011;28:485-93.

30. Adjei Boakye E, Tobo BB, Rojek RP, Mohammed KA, Geneus CJ, Osazuwa-Peters N. Approaching a decade since HPV vaccine licensure: racial and gender disparities in knowledge and awareness of HPV and HPV vaccine. Hum Vaccines Immunother. 2017;13:2713-22.

31. Gilkey MB, McRee A-L. Provider communication about HPV vaccination: a systematic review. Hum Vaccines Immunother. 2016;12:1454-68.

32. De Alba I, Sweningson JM. English proficiency and physicians' recommendation of Pap smears among Hispanics. Cancer Detect Prev. 2006;30:292-6.

33. Polonijo AN, Carpiano RM. Social inequalities in adolescent human papillomavirus (HPV) vaccination: a test of fundamental cause theory. Soc Sci Med. 2013;82:115-25.

34. Harlan LC, Bernstein AB, Kessler LG. Cervical cancer screening: who is not screened and why? Am J Public Health. 1991;81:885-90.

35. Rosenthal SL, Weiss TW, Zimet GD, Ma L, Good MB, Vichnin MD. Predictors of HPV vaccine uptake among women aged 19-26: importance of a physician's recommendation. Vaccine. 2011;29:890-5.

36. Gilkey MB, Calo WA, Moss JL, Shah PD, Marciniak MW, Brewer NT. Provider communication and HPV vaccination: the impact of recommendation quality. Vaccine. 2016;34:1187-92.

37. Lau M, Lin $\mathrm{H}$, Flores $\mathrm{G}$. Factors associated with human papillomavirus vaccine-series initiation and healthcare provider recommendation in US adolescent females: 2007 National Survey of Children's Health. Vaccine. 2012;30:3112-8.

38. Thompson EL, Galvin AM, Daley EM, Tatar O, Zimet GD, Rosberger Z. Recent changes in cervical cancer screening guidelines: US women's willingness for HPV testing instead of Pap testing. Prev Med. 2020;130: 105928.

39. CDC. BRFSS Web Enabled Analysis Tool (WEAT). https://nccd.cdc.gov/weat/. Accessed 28 Sep 2021.

40. NORC at the University of Chicago. Technical overview of the Amerispeak ${ }^{\circledast}$ Panel: Norc's Probability-Based Household Panel. 2021. https:// amerispeak.norc.org/Documents/Research/AmeriSpeak\%20Technical\%200verview\%202019\%2002\%2018.pdf.

41. CDC. BRFSS statistical brief on cervical cancer screening questions. https://www.cdc.gov/brfss/data_documentation/pdf/brfss-statistical-brief-cervical-cancer-screening-questions-508.pdf.

42. Casper MJ, Clarke AE. Making the Pap smear into the "right tool" for the job: cervical cancer screening in the USA, circa 1940-95. Soc Stud Sci. 1998;28:255-90.

43. National Committee for Quality Assurance, O'Kane M, Agrawal S, Anthem, Inc., Binder L, The Leapfrog Group, et al. An equity agenda for the field of health care quality improvement. NAM Perspect. 2021;11. https://nam.edu/an-equity-agenda-for-the-field-of-health-carequality-improvement. Accessed 9 Nov 2021.

44. Boersma P, Black L. Human papillomavirus vaccination among adults. 2020;

45. Goodman K, Black CM. Patient knowledge of STI testing in an urban clinic. J Am Acad Phys Assist. 2018;31:36-41.

46. Adegoke O, Kulasingam S, Virnig B. Cervical cancer trends in the United States: a 35-year population-based analysis. J Womens Health. 2012;21:1031-7.

47. Benard VB, Watson M, Saraiya M, Harewood R, Townsend JS, Stroup AM, et al. Cervical cancer survival in the United States by race and stage (2001-2009): findings from the CONCORD-2 study. Cancer. 2017;123:5119-37.

48. Gearhart PA, Randall TC. Human papillomavirus (HPV) workup: approach considerations, cytologic testing, HPV DNA Typing. Medscape; 2020. https://emedicine.medscape.com/article/219110-workup\#c8.

49. Deftereos G. Human papillomavirus (HPV) testing - cervical cancer screening. ARUP Consult; 2021. https://arupconsult.com/content/ human-papillomavirus.

50. CDC. Genital HPV infection-CDC Fact Sheet. 3

51. NIH. HPV and Cancer-National Cancer Institute. 2019. https://www.cancer.gov/about-cancer/causes-prevention/risk/infectious-agents/ hpv-and-cancer. Accessed 9 Nov 2021.

Publisher's Note Springer Nature remains neutral with regard to jurisdictional claims in published maps and institutional affiliations. 